\title{
Switching Capacities based Envelope Amplifier for High Efficiency RF Amplifiers
}

\author{
M.Vasić, O. García, J.A. Oliver, P. Alou, D. Diaz, J.A. Cobos \\ Centro de Electrónica Industrial \\ Universidad Politécnica de Madrid \\ Madrid, Spain \\ miroslav.vasic@upm.es
}

\begin{abstract}
Modern transmitters usually have to amplify and transmit signals with simultaneous envelope and phase modulation. Due to this property of the transmitted signal, linear power amplifiers (class A, B or AB) are usually used as a solution for the power amplifier stage. These amplifiers have high linearity, but suffer from low efficiency when the transmitted signal has high peak-to-average power ratio (PAPR). The Kahn envelope elimination and restoration (EER) technique is used to enhance efficiency of RF transmitters, by combining highly efficient, nonlinear RF amplifier (class E) with a highly efficient envelope amplifier in order to obtain linear and highly efficient RF amplifier. This paper presents a solution for the envelope amplifier based on a multilevel converter in series with a linear regulator. The multilevel converter is implemented by employing voltage dividers based on switching capacities. The implemented envelope amplifier can reproduce any signal with maximum spectral component of $2 \mathrm{MHz}$ and give instantaneous maximum power of $50 \mathrm{~W}$. The efficiency measurements show that when the signals with low average value are transmitted, the implemented prototypes have up to $20 \%$ higher efficiency than linear regulator that is used as a conventional solution.
\end{abstract}

\section{INTRODUCTION}

In the modern radio communications it is necessary to transmit as much data as possible for the given bandwidth. The best signal modulations are those that perform simultaneous phase and amplitude modulation, and therefore the linearity of the power amplifiers that are used in these systems is essential. Due to this demand, linear power amplifiers, such as class A or class B, are employed, but, unfortunately, they suffer from extremely poor efficiency when the transmitted signal has low values. For example, ideal class A and class B amplifiers have average efficiency of only $5 \%$ and $28 \%$, respectively, when signals with Rayleigh's envelope distribution are transmitted [1]. One of the techniques that are used to enhance the efficiency of the power amplifiers is the Kahn's technique. The Kahn's envelope estimation and restoration (EER) technique proposes use of dc-dc converter (envelope amplifier) that should modulate the voltage supply of a highly efficient but nonlinear power amplifier (class E or class D) [2], Figure 1. This idea is based on the fact that any narrow band signal can be presented as simultaneous amplitude (envelope) and phase modulation:

$$
\begin{array}{r}
V_{R F}(t)=I(t) \cos (2 \pi f t)-Q(t) \sin (2 \pi f t)= \\
=A(t) \cos (2 \pi f t+\theta(t)) \\
\theta(\mathrm{t})=\operatorname{arctg}\left(\frac{Q(t)}{I(t)}\right) \quad A(t)=\sqrt{I(t)^{2}+Q(t)^{2}}
\end{array}
$$

where $f$ is the carrier frequency, $Q(t)$ and $I(t)$ are modulated signals.

In the state of the art, several solutions for the envelope amplifier can be found, such as a simple buck converter (class $\mathrm{S}$ modulator) in [3-5], multiphase buck converter in [6], threelevel converter in [7] or linear assisted switching amplifier [8, 9]. These solutions do not exceed the bandwidth of few hundred $\mathrm{kHz}$ and the output power is from the range of $\mathrm{mW}$ up to several tens of watts. Their use for applications that require bandwidth in the $\mathrm{MHz}$ range is limited, for example, a buck converter with $1 \mathrm{MHz}$ bandwidth should have switching frequency of, at least, $5 \mathrm{MHz}$.

The envelope amplifier should have high linearity, fast dynamic response, high efficiency and small interference with the spectrum of the output signal. Regarding all these restrictions, a solution that is based on a multilevel converter in series with a high slew rate linear regulator is presented in [10], Figure 2. The multilevel converter in this paper is implemented by voltage dividers based on switching capacities. Converters that employ switching capacities offer high efficiency and do not need any bulky magnetic component, thus their size is significantly smaller comparing them with classical converters and gives a possibility for integration [11]. The implemented envelope amplifier can reproduce a sine wave or any other reference of $2 \mathrm{MHz}$, and give the maximum power of $50 \mathrm{~W}$. Commercial (or academic) solutions to obtain this bandwidth for the envelope amplifier are based on a linear regulator. Unfortunately, this means low efficiency for the envelope amplifier, especially when the output signal has low voltage levels. 


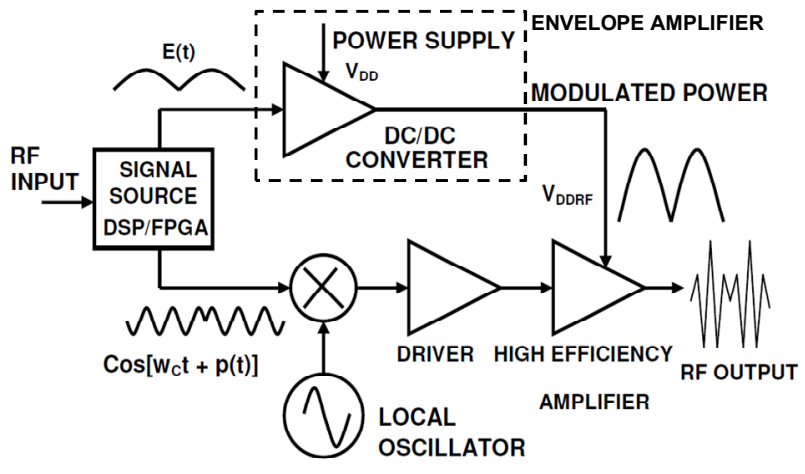

Figure 1. Block Scheme of Kahn-technique Transmitter

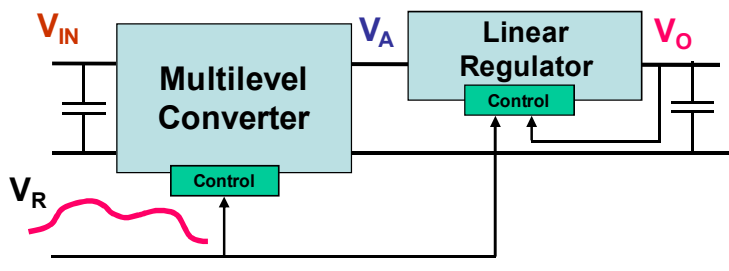

Figure 2. Simplified schematic of the proposed envelope amplifier

\section{PROPOSED SOLUTION}

The multilevel converter has to supply the linear regulator and it has to provide discrete voltage levels that are as close as possible to the output voltage of the envelope amplifier. If this is fulfilled, the power losses on the linear regulator will be minimal, because they are directly proportional to the difference of its input and output voltage. However, in order to guarantee correct work of the linear regulator, the output voltage of the multilevel converter always has to be higher than the output voltage of the linear regulator. Time diagrams of the multilevel converter and linear regulator voltage are shown in Figure 3.

The linear regulator can be designed to have very high bandwidth, and it should filter all the noise that could come from the multilevel converter. Therefore, the multilevel converter does not need any filter at its output and the design of the complicated filter as in the case of switched converters is avoided.

The multilevel converter presented in [10] is based on independent voltage cells that are put in series and turned on and off depending on the level of the sent reference. The multilevel solution in this paper is proposed in [10] and it is based on the independent voltage sources that supply the linear regulator through an analog multiplexer. The selection of the active voltage source will depend on the level of the envelope reference. A simplified schematic of the multilevel converter is presented in Figure 4.

The efficiency of the linear regulator depends on the number of the voltage levels that are applied and their distribution. In [10] it is shown that optimizing voltage levels leads to significant increase of efficiency comparing it with the solution with equidistant voltage levels. Due to the tradeoff between the complexity of the layout, PCB parasitic and possible system efficiency, three voltage levels are selected as an efficient and feasible solution. The optimal distribution for three voltage levels when a signal with high PAPR is transmitted is approximately: $\mathrm{V}_{\mathrm{MAX}}, 3 / 4 \mathrm{~V}_{\mathrm{MAX}}, 1 / 2 \mathrm{~V}_{\mathrm{MAX}}$, where $\mathrm{V}_{\mathrm{MAX}}$ is the maximum level of the output signal's envelope. Due to convenient voltage distribution, these three voltage levels can be produced by two voltage dividers that are based on switching capacities. The input terminals of the first voltage divider should be connected to $\mathrm{V}_{\mathrm{MAX}}$ and ground and the voltage at its output would be $1 / 2 V_{\text {MAX }}$. The input terminals of the second voltage divider should be connected to $\mathrm{V}_{\mathrm{MAX}}$ and $1 / 2 \mathrm{~V}_{\mathrm{MAX}}$. Its output voltage would be $1 / 4 \mathrm{~V}_{\mathrm{MAX}}$ referring it to the output of the first voltage divider, i.e. $3 / 4 \mathrm{~V}_{\mathrm{MAX}}$ referring it to the ground of the system. Both voltage dividers are implemented in the same way, using the same topology presented in [11] and shown in Figure 5.

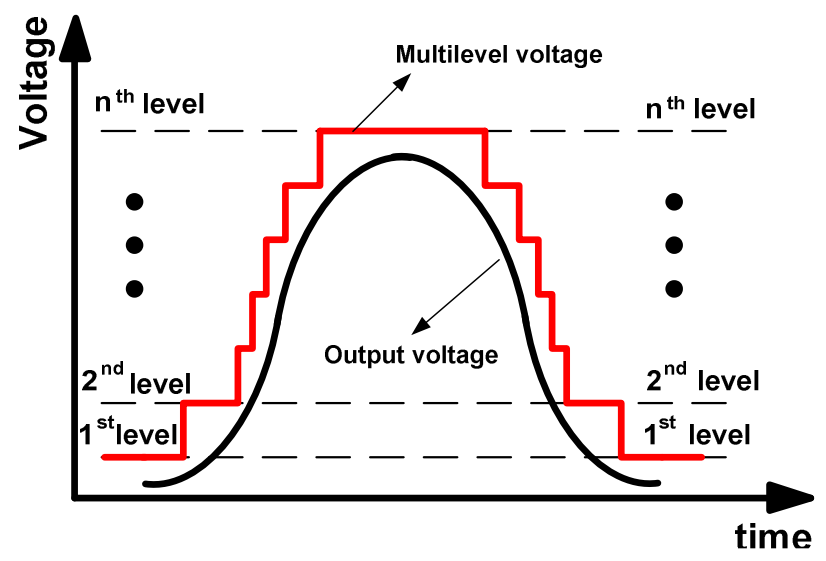

Figure 3. Time diagrams of the proposed envelope amplifier

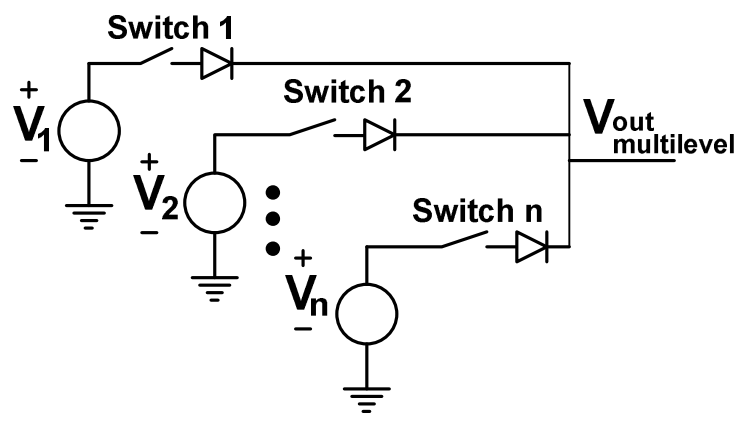

Figure 4. Multilevel converter realized with independent supplies and analog multiplexer

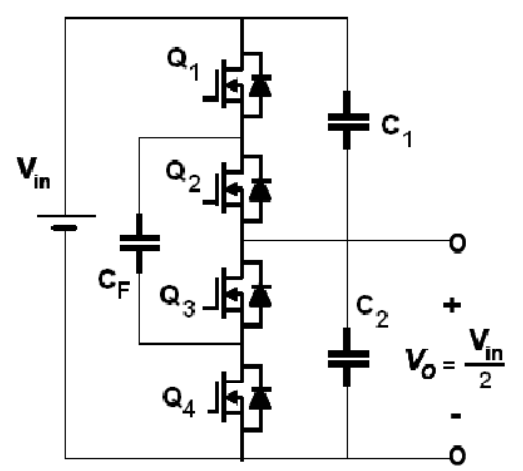

Figure 5. Voltage divider based on switching capacities 
Solutions based on switching capacities offer high efficiency at very wide load profiles and low switching frequencies. Additionally, this topology does not use any inductive element and, therefore, are convenient for integration. This is significant improvement comparing proposed solution with the multilevel solution in [10], where it was necessary to implement a flyback converter with three outputs, and where the used transformer was a very bulky, low-efficient component. The possible problems in this solution are increased switching noise due to lack of filtering and complex designs that are used for closed loop solutions. In the proposed solutions the voltage dividers do not need to have precise control of its output voltages, because the linear regulator that is put in series will perform the fine regulation, therefore, the voltage dividers can work in open loop, while the linear regulator should filter all the switching noise that comes from the voltage dividers.

The only part of the system that uses high switching frequency is the analog multiplexer. If the sent reference is a 2 $\mathrm{MHz}$ sine wave, the MOSFET's switching frequency inside the multiplexer will be $2 \mathrm{MHz}$ as well. Therefore, it can be said that even in the cases when the reference is a high frequency signal, the maximum switching frequency in the system is relatively low.

\section{DESIGNED SYSTEM}

In order to prove the concept a prototype of envelope amplifier has been made. The specifications of the envelope amplifier prototype are as follows:

\section{- The input voltage is $24 \mathrm{~V}$}

- $\quad$ The output voltage can be changed from $0 \mathrm{~V}$ to $24 \mathrm{~V}$

- The maximum power of the prototype is $50 \mathrm{~W}$

- The maximum frequency of the reference signal is $2 \mathrm{MHz}$

As it is aforementioned, the voltage dividers will work in open loop, and, in order to guarantee stable voltages at their outputs, at each output there are two ceramic capacitors in parallel (each one of $22 \mathrm{uF}$ ). The power losses in the voltage divider can be divider in two parts. The first part represents the power losses due to parasitic capacitance between the gate and source of the used MOSFETs, while the second part of the losses is due to finite resistance of the used switches and voltage difference between the flying capacitor and output capacitors. The gate losses are directly proportional to the switching frequency of the voltage divider. In order to conclude how the power losses due to the second mechanism depend on the switching frequency, it is necessary to conduct the analysis presented in [12]. It can be shown that theses losses can be modeled with output resistance of the voltage divider that is equal to:

$$
R_{\text {out }}=\frac{1}{C_{F} f_{s w}}
$$

where $C_{F}$ is the value of the "flying" capacitor and $f_{S W}$ is the switching frequency of the converter. Therefore, for low power losses it is necessary to apply low switching frequency and use flying capacitor as big as possible (in this design there are five $22 \mathrm{uF}$ ceramic capacitors in parallel). The switching frequency for both dividers is $50 \mathrm{kHz}$. After the voltage dividers have been implemented, the efficiency of $12 \mathrm{~V}$ and $18 \mathrm{~V}$ outputs has been measured. The result of the measurement is shown in Figure 6. It can be seen that the efficiency of both voltage dividers is higher than $95 \%$ in very wide range of output power.

As it is explained in [11], the drivers that are used in order to govern the employed MOSFETS are supplied by the voltage that is equal to the half of the voltage divider's input voltage. The input terminals of one driver are connected between the ground and the output terminal of the voltage divider, while the input terminals of the second driver are connected between the input and output voltage. Having this in mind, in the first voltage divider the used driver has to be able to support $1 / 2$ $\mathrm{V}_{\mathrm{MAX}}$ as its supply voltage and in the second divider the maximum voltage supply is $1 / 4 \mathrm{~V}_{\mathrm{MAX}}$. In the first voltage divider the used drivers are IR2181 and in the second LM27222. The MOSFETs are the same in both voltage dividers and they are Si4864. The analog multiplexer is implemented using MOSFETs and diodes like in Figure 7.

The diodes are necessary in order to prevent current flow between the voltage sources due to parasitic diodes of the MOSFETs. As it can be seen, the MOSFETs from the analog multiplexer it have "floating" source and it is necessary to use additional power sources and isolation chips in order to control them, Figure 8. The isolation chips are ISO721 and the drivers are EL7156.

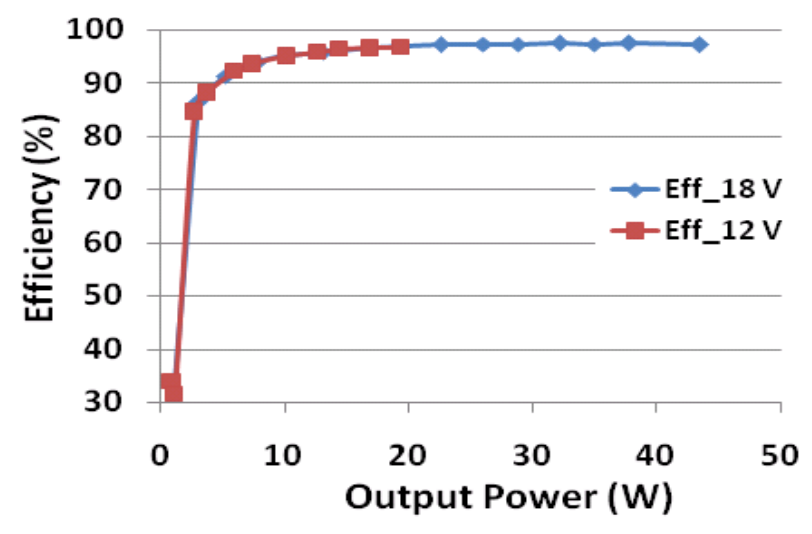

Figure 6. Measured efficiency of the implemented multilevel converter

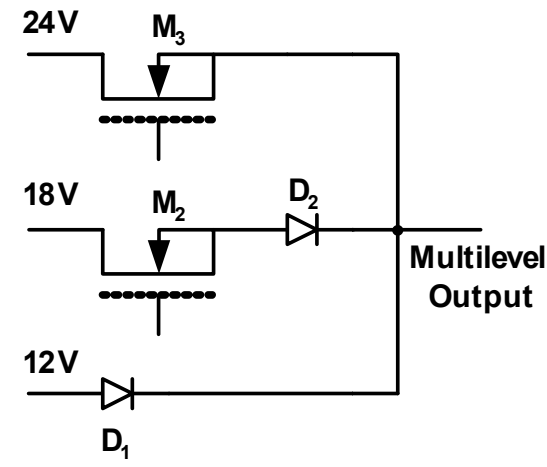

Figure 7. Implemented analog multiplexer 


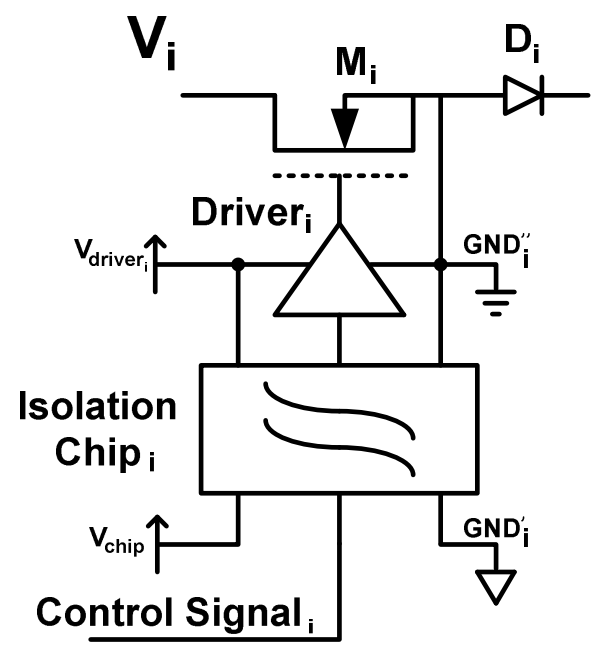

Figure 8. Simplified schematic of the circuit that is used to govern the MOSFETs in the implemented analog multiplexer

The linear regulator that is used as the last stage of the envelope amplifier should have high bandwidth and the components are selected in order to accomplish this request. The MOSFET that is used as a pass element for the linear regulator (BLF 177) is from HF/VHF power MOS family of transistors. The operational amplifier is LM6172 and it is selected because of its high bandwidth. The systems schematic is shown in Figure 9.

The triggering logic is implemented in a FPGA that is used as a source of the digital signal reference. The digitalized signal reference is sent to a D/A converter and from there to the linear regulator. The same reference signal is sent to the triggering logic and to the linear regulator, but it is of the most importance that the reference that the linear regulator receives is synchronized with the output voltage of the multilevel converter. Only when these two voltages are synchronized, the system's output voltage will be always lower than the output voltage of the multilevel converter. Therefore, a digital delay filter is implemented in the FPGA as well, in order to compensate the delays in the system and synchronize the multilevel output voltage with linear regulator's reference. The load of the system is a $10 \Omega$ resistance.

Figure 10 shows a photograph of the implemented envelope amplifier.

\section{EXPERIMENTAL RESULTS}

In order to characterize the envelope amplifier, it has been tested with different sine waves. In table 1, the efficiency of the implemented envelope amplifier is shown depending on the frequency of the reproduced sine wave and its dc offset and amplitude. In the same table, the measured efficiency is compared with the efficiency of the ideal linear regulator and with the efficiency of the envelope amplifier that is implemented with independent voltage cells [10]. As it can be seen, the efficiency of the hybrid solution has up to $20 \%$ better efficiency than an ideal linear amplifier when a transmitted signal has low average values, and that is mostly the case when the EER technique is applied. It is important to note that the envelope amplifier based on the switching capacities has better efficiency than the envelope amplifier based on independent voltage cells [10]. Figures 11 and 12 show the multilevel and system's output voltage in the case when a reference is a sine wave of $500 \mathrm{kHz}$ and $2 \mathrm{MHz}$ respectively.

TABLE I. MEASURED EFFICIENCY OF THE IMPLEMENTED ENVELOPE AMPLIFIER FOR DIFFERENT SINE WAVES COMPARED WITH THE THEORETICAL EFFICIENCY OF AN IDEAL LINEAR REGULATOR SUPPLIED BY $23 \mathrm{~V}$

\begin{tabular}{|c|c|c|c|c|}
\hline Vsin(V) & $\begin{array}{c}\text { Sine wave } \\
\text { frequency } \\
\text { (MHz) }\end{array}$ & $\begin{array}{c}\text { Measure } \\
\text { efficiency of } \\
\text { the envelope } \\
\text { amplifier } \\
\text { based on } \\
\text { switching } \\
\text { capacities }\end{array}$ & $\begin{array}{c}\text { Measure } \\
\text { efficiency of } \\
\text { the envelope } \\
\text { amplifier } \\
\text { based on the } \\
\text { independent } \\
\text { voltage cells }\end{array}$ & $\begin{array}{c}\text { Theoretical } \\
\text { efficiency } \\
\text { of an ideal } \\
\text { linear } \\
\text { regulator } \\
\text { supplied } \\
\text { by 23V }\end{array}$ \\
\hline $0-9$ & 0.5 & $47.5 \%$ & $43.3 \%$ & $29.3 \%$ \\
\hline $5-14$ & 0.5 & $61.5 \%$ & $58.9 \%$ & $45.9 \%$ \\
\hline $0-22.5$ & 0.5 & $75.2 \%$ & $69.7 \%$ & $73.0 \%$ \\
\hline $0-9$ & 2 & $48.2 \%$ & $43.7 \%$ & $28.1 \%$ \\
\hline $5-14$ & 2 & $59.0 \%$ & $58.8 \%$ & $45.9 \%$ \\
\hline $0-22.5$ & 2 & $70.9 \%$ & $68.3 \%$ & $73.0 \%$ \\
\hline
\end{tabular}

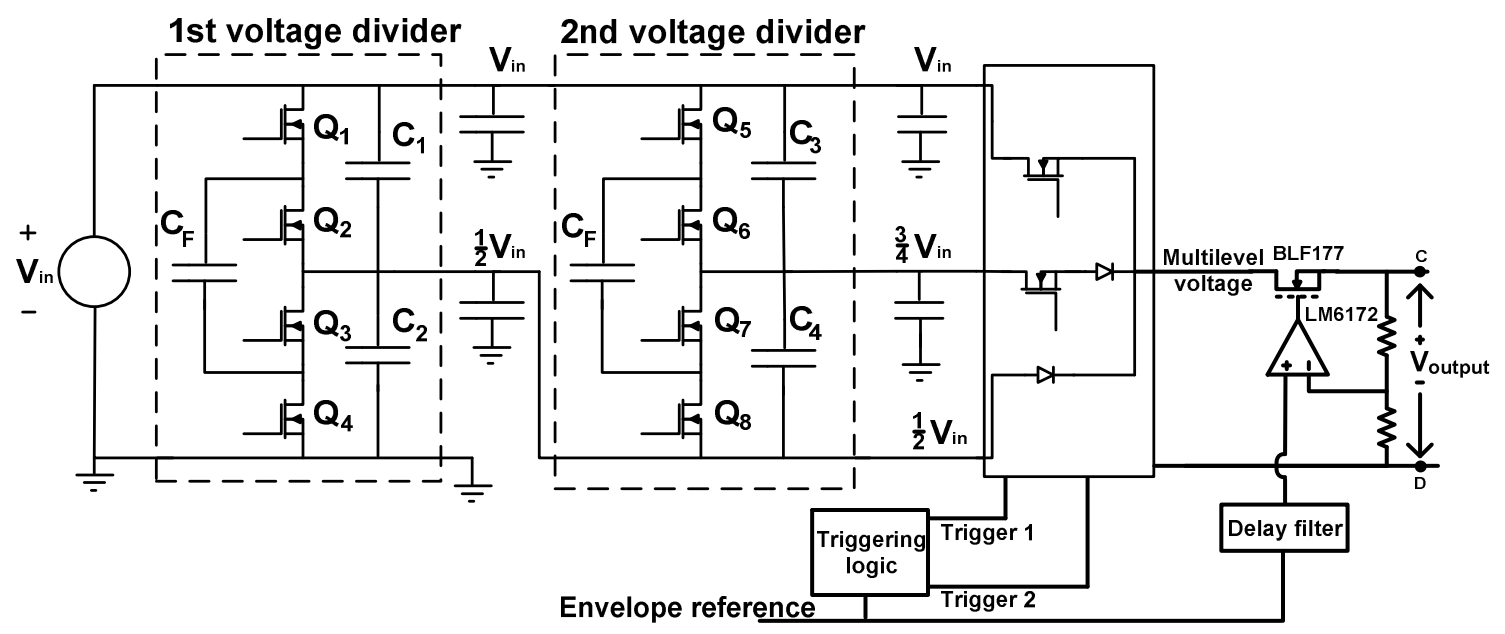

Figure 9. Simplified schematic of the implemented envelope amplifier 


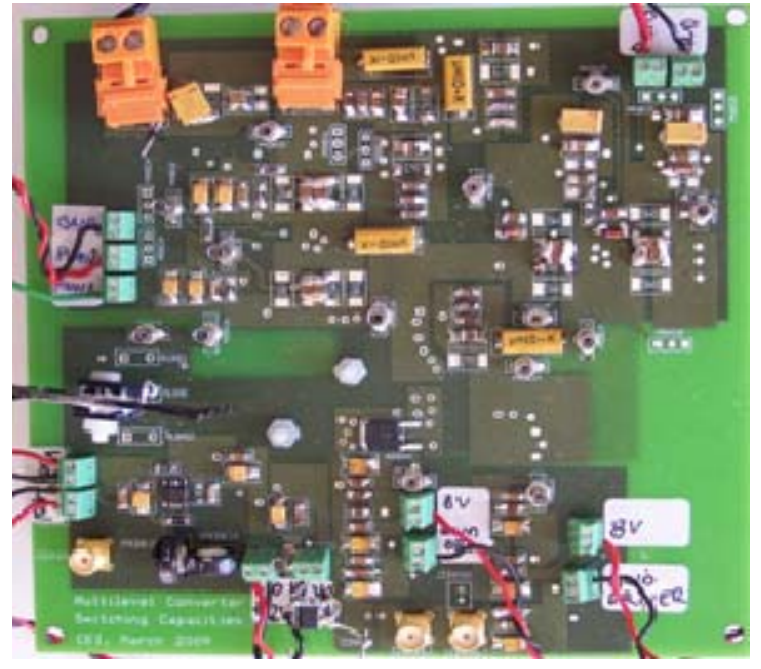

Figure 10. Photograph of the implemented envelope amplifier

The linearity and the bandwidth of the envelope amplifier are crucial in order to obtain high linearity of the power amplifier based on EER technique, therefore, these measurements have been performed as well. The linearity measurements are conducted by two tone tests, where two sine waves of the same amplitude are used as a reference signal and at the output of the envelope amplifier the ratio between the reproduced amplitudes and the intermodulation harmonics that are produced by the envelope amplifier is observed. Figure 13 shows spectral content at the output of the implemented envelope amplifier in the case when a two tone signal composed of $1 \mathrm{MHz}$ and $1.05 \mathrm{MHz}$ is used. It can be observed that the attenuation of the intremodulation components is higher than $50 \mathrm{~dB}$, what means high linearity of the envelope amplifier.

In [1] it has been explained that the bandwidth of the envelope amplifier has to be, at least, two times higher than the bandwidth of the RF signal. The reproduced envelope should not have any attenuation up to $2 \mathrm{MHz}$ and it has been shown that the proposed envelope amplifier can reproduce 2 $\mathrm{MHz}$ sine wave of maximum amplitude. However, this does not mean that the implemented envelope amplifier cannot reproduce higher harmonics. The higher harmonics that are very important for high linearity of Kahn's transmitter usually are of much smaller amplitudes than the maximum amplitude that can be reproduced by the envelope amplifier. Based on the analysis presented in [1], a test with rectified sine wave has been conducted. If the reference signal is a rectified sine wave of frequency $f$, its spectrum is infinite and consists of tones that are placed on frequencies $2 f, 4 f$, $6 f$... A rectified 500 $\mathrm{kHz}$ sine wave of maximum amplitude has been used as the reference and the response of the envelope amplifier has been measured, Figure 14. The spectrum of the output signal is compared with the spectrum of the reference signal, Figure 15. It can be seen that the proposed envelope amplifier admits even the harmonic higher than $2 \mathrm{MHz}$.

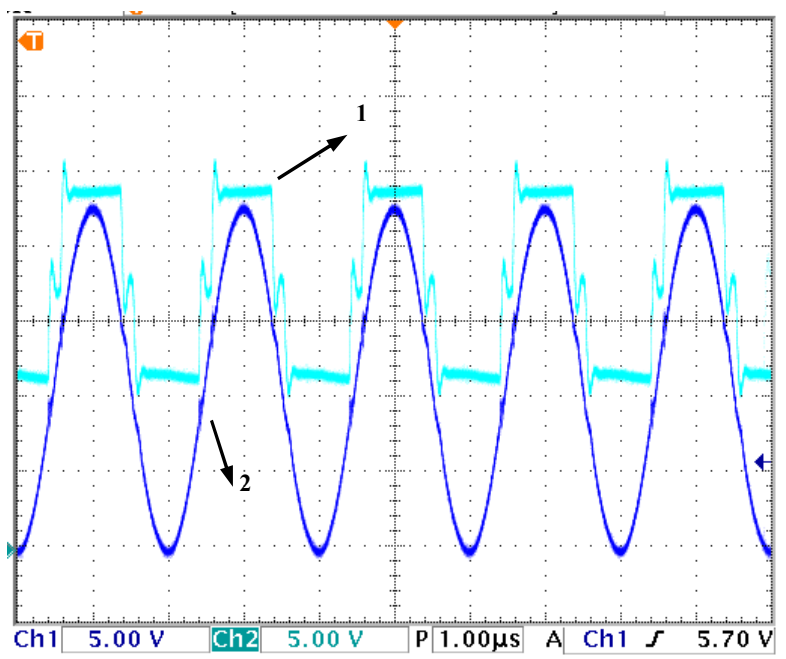

Figure 11. Multilevel output voltage (label 1) and envelope amplifier's output voltage (label 2) in the case of a $500 \mathrm{kHz}$ sine wave

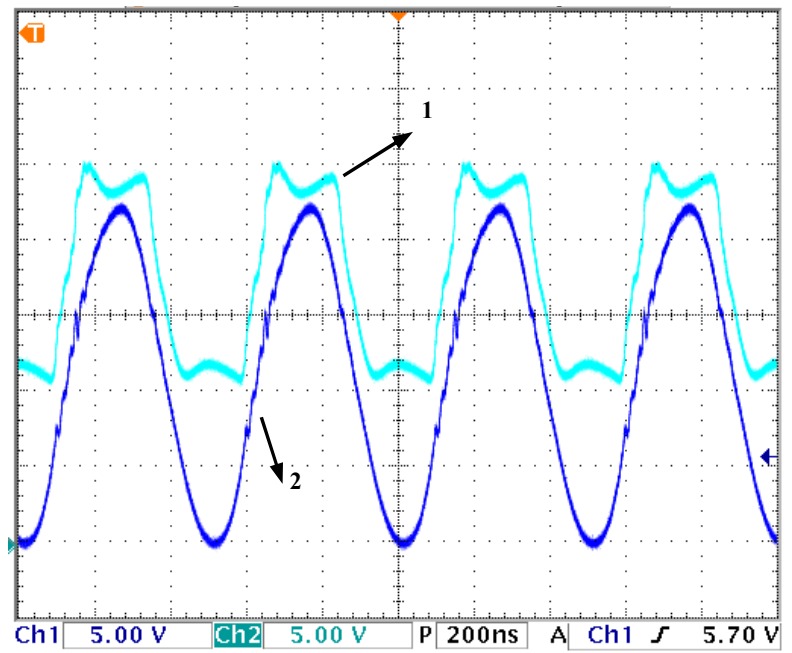

Figure 12. Multilevel output voltage (label 1) and envelope amplifier's output voltage (label 2) in the case of a $2 \mathrm{MHz}$ sine wave

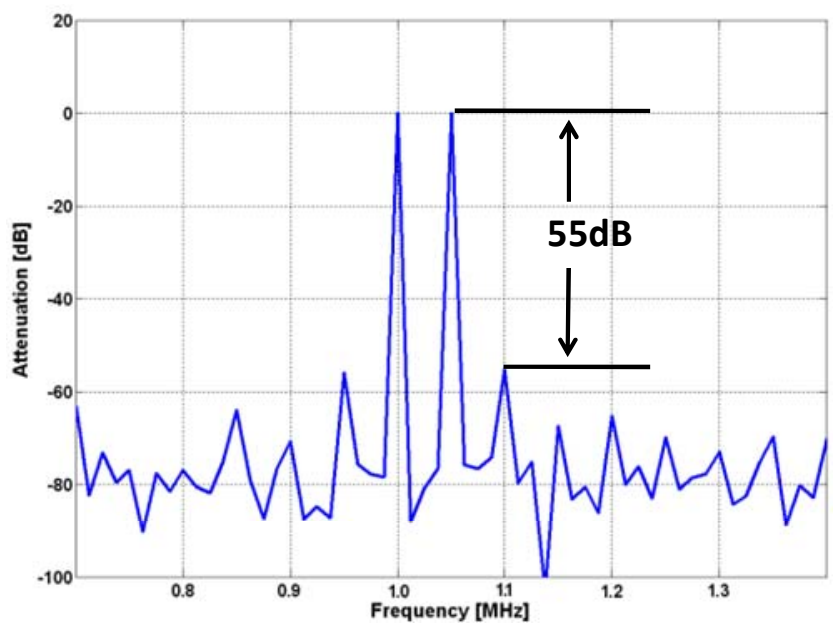

Figure 13. Attenuation of the intermodulation harmonics in the case when a reference signal is a two tone signal composed of $1 \mathrm{MHz}$ and $1.05 \mathrm{MHz}$ sine waves. The measurement is processed in MATLAB 


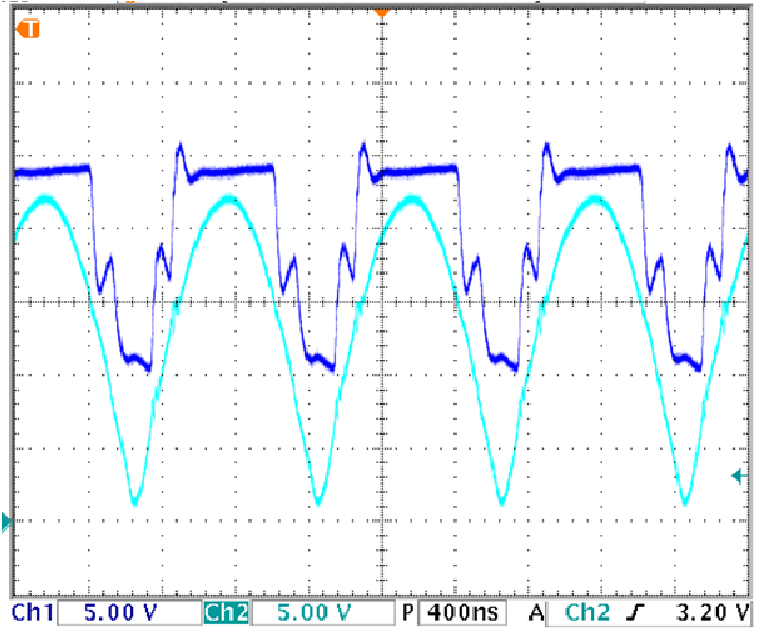

Figure 14. Waveform of multilevel's output voltage and linear regulator's output voltage when a rectified $500 \mathrm{kHz}$ sine wave is used as the reference

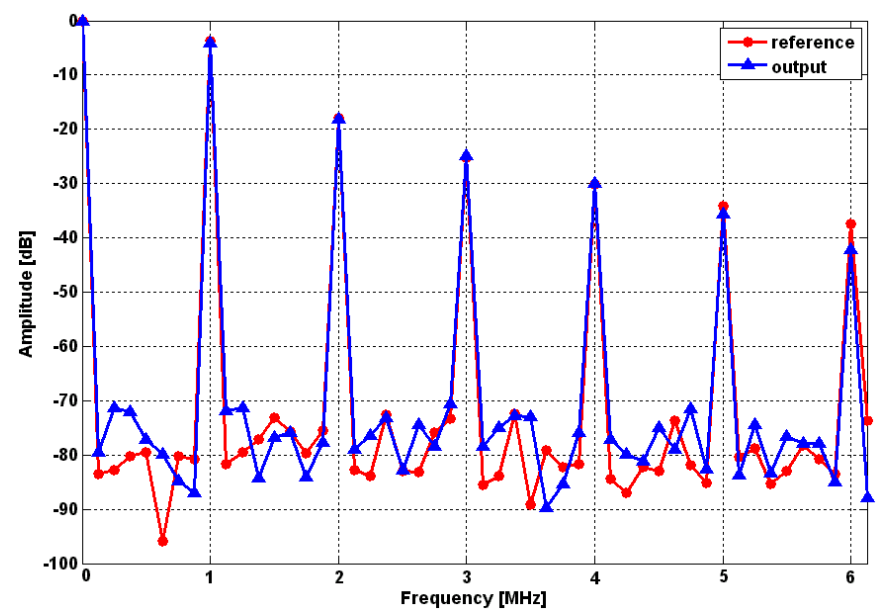

Figure 15. Spectrum of the reference and output signal when a rectified 500 $\mathrm{kHz}$ sine wave is used as the reference. All the values are scaled to the dc value of the signal

\section{CONCLUSIONS}

In this paper a solution for envelope amplifier in EER technique is presented. The solution consists of a multilevel converter in series with a linear regulator. The proposed multilevel converter is implemented by voltage dividers based on switching capacities. The designed envelope amplifier can provide up to $50 \mathrm{~W}$ of instantaneous power and reproduce a sine wave up to $2 \mathrm{MHz}$. The switching frequency of voltage dividers is low $(50 \mathrm{kHz})$ comparing it to the bandwidth of the envelope amplifier. Therefore the proposed solution uses lower switching frequency than a conventional dc-dc converter for the same given bandwidth.

The multilevel voltage levels are selected in order to maximize the efficiency of the linear regulator for the signals with high PAPR. The efficiency of the envelope amplifier has been measured for different sine waves and its efficiency is up to $20 \%$ higher than in the case of an ideal linear regulator when sine waves have low average value (what is usually the case in the RF systems). The linearity of the prototype is measured as well and the attenuation of the intermodulation harmonics is higher than $50 \mathrm{~dB}$. The implemented prototype does not have any magnetic component and it can be integrated.

\section{REFERENCES}

[1] F.H. Raab, "Intermodulation Distortion in Kahn-Technique Transmitters", IEEE Transactions on Microwave Theory and Techniques, Volume 44, Issue 12, Part 1, Decemeber 1996, Pages: 2273-2278

[2] F.H. Raab, P. Asbeck, S. Cripps, P.B. Kenington, Z.B. Popovic, N Pothecary, J.F. Sevic, N.O. Sokal, "Power amplifiers and transmitters for RF and microwave," IEEE Trans. on Microwave Theory and Techniques, Volume: 50, Issue: 3, March 2002, Pages: 814-826.

[3] P. Midya, K. Haddad, L. Connell, S. Bergstedt, B. Roeckner, "Tracking power converter for supply modulation of RF power amplifiers," IEEE Power Electronics Specialists Conference, PESC. 2001, Vol. 3, Pages: $1540-1545$

[4] J. Staudinger, B. Gilsdorf, D. Newman, G. Norris, G. Sadowniczak, R. Sherman, T. Quach, "High efficiency CDMA RF power amplifier using dynamic envelope tracking technique," Microwave Symposium Digest., IEEE MTT-S International, Vol. 2, June 2000, Pages: 873-876

[5] M.C.W. Hoyerby, M.A.E. Andersen, "Envelope tracking power supply with fully controlled 4th order output filter", Power Electronics Conference, APEC '06, March. 2006

[6] A. Soto, J.A. Oliver, J.A. Cobos, J. Cezon, F. Arevalo, "Power supply for a radio transmitter with modulated supply voltage", Applied Power Electronics Conference, APEC '04, Volume: 1, Feb. 2004 Pages:392 398

[7] V. Yousefzadeh, E. Alarcon, D. Maksimović, "Three-level buck converter for envelope tracking in RF power amplifiers," IEEE Trans. on Power Electronics, Volume:21, Issue: 2, March 2006, Pages:549 552

[8] US Patent No. 6084468, Method and Apparatus for High Efficiency Wideband Power Amplification, July 2000.

[9] V. Yousefzadeh, E. Alarcon, D. Maksimović, "Efficiency optimization in linear assisted switching power converters for envelope tracking in RF power amplifiers", IEEE International Symposium on Circuits and Systems, ISCAS 2005, 23-26 May, pages:1302-1305 Vol. 2

[10] M.Vasić, O.Garcia, J.A.Oliver, P.Alou, D.Diaz, J.A.Cobos, "Multilevel Power Supply for High Efficiency RF Amplifier", Proc. of the 24th Annual IEEE Applied Power Electronics Conference, APEC '09, February 2009

[11] J.Sun, M.Xu, Y.Ying, F.C.Lee, "High Power Density, High, Efficiency System Two-stagePower Architecture for Laptop Computers", 37th IEEE Power Electronics Specialists Conference / June 18 - 22, 2006, Jeju, Korea

[12] S. Ben-Yaakov, "Switched Capacitors Converters", Professional Education Seminar at the 24th Annual IEEE Applied Power Electronics Conference, APEC '09, February 2009 